\title{
Le plurilinguisme dans les productions audiovisuelles pour la diffusion et la vulgarisation de la science et de la technologie
}

\section{Multilingualism in audiovisual productions for the dissemination and popularization of science and technology}

\section{El plurilingüismo en las producciones audiovisuales para la difusión y popularización de la ciencia y la tecnología}

https://doi.org/10.52358/mm.vi7.206

Teurra Fernandes Vailatti, doctorante

Programa de Pós Graduação em Letras, Universidade Federal do Paraná, Brésil

Laboratoire de Linguistique et Didactique des Langues Étrangères et Maternelles, Université Grenoble

Alpes, France

teurravailatti@hotmail.com

RÉSUMÉ

Dans ce travail, nous nous focalisons sur le rôle du plurilinguisme dans une capsule vidéo pour la diffusion et la vulgarisation d'une recherche scientifique dans le cadre d'un événement promu par une université brésilienne. Devant l'usage privilégié du portugais (langue nationale) et de l'hégémonie de l'anglais dans la production scientifique, nous montrons comment le 
plurilinguisme peut valoriser la recherche en s'intégrant dans la communication scientifique et nous discutons de son importance pour une construction plurielle des savoirs. Le compte rendu de cette expérience a pour but de contribuer à l'amélioration des pratiques communicatives de l'enseignement supérieur qui visent à atteindre un public non spécialisé.

Mots-clés : plurilinguisme, recherche scientifique, audiovisuel, vulgarisation de la science, internationalisation de l'université

\section{ABSTRACT}

This study focuses on the role of multilingualism in a video capsule for the dissemination and popularization of scientific research within the framework of an event promoted by a Brazilian university. Faced with the privileged use of Portuguese (the national language) and the hegemony of English in scientific production, we show how multilingualism can enhance research by integrating scientific communication and discussing its importance for a plural construction of knowledge. This experience report aims to improve communicative practices in higher education to reach a non-specialist audience.

Keywords: multilingualism, scientific research, audiovisual, popularization of science, university internationalization

\section{RESUMEN}

Este trabajo consiste en analizar el plurilingüismo de una cápsula de vídeo para la difusión y divulgación de la investigación científica en el contexto de un evento promovido por una universidad brasileña. Frente al uso privilegiado del portugués (lengua nacional) y la hegemonía del inglés en la producción científica, mostramos cómo el plurilingüismo puede potenciar la investigación al integrarse en la comunicación científica y discutimos su importancia para una construcción plural del conocimiento. El propósito de este estudio de experiencia es contribuir a la mejora de las prácticas comunicativas en la educación superior que pretenden llegar a un público no especializado.

Palabras clave: plurilingüismo, investigación científica, audiovisual, divulgación de la ciencia, internacionalización de la universidad 


\section{Introduction}

Ce travail se fonde sur une étude personnelle' portant sur l'analyse d'une capsule vidéo élaborée, par l'auteure, dans le cadre d'un événement promu par le Programa de Pós-Graduação em Letras ${ }^{2}$ de I'Université Fédérale du Paraná (PPGL-UFPR), au Brésil. L'événement « Minha pesquisa em 5 minutos ${ }^{3}$ » consiste à publier en ligne des vidéos d'environ cinq minutes, produites par les étudiants du PPGL, dans un format préétabli, dans le but de présenter au public en général (non spécialisé) une synthèse des recherches de master/doctorat en linguistique et en littérature. Les vidéos sont publiées sur la chaîne YouTube et sur le compte Instagram du PPGL-UFPR et peuvent être diffusées par le programme sur d'autres plateformes, telles que Facebook, et par courriel. II s'agit d'un événement consacré à la diffusion et à la vulgarisation de la science et de la technologie et qui est, par conséquent, guidé par un registre de langue et un format accessibles, par opposition à un format et un langage universitaires.

Nous réalisons ainsi une analyse de notre production en nous interrogeant sur la façon dont le plurilinguisme peut valoriser la recherche dans un événement scientifique. Sur le plan méthodologique, nous proposons une approche d'analyse qualitative et interprétative, dans une perspective focalisée sur les concepts d'énoncé multimodal (Bakhtin, 1992; Rojo, 2013) et de paysage linguistique (PL) (Gorter, 2006; Gorter, Shohamy, 2009; Melo-Pfeifer, Lima-Hernandes, 2020), avec un intérêt particulier pour la dimension plurilingue de la capsule vidéo, c'est-à-dire les rapports entre les langues pour la construction d'un énoncé global. En se basant sur cette analyse, nous repérons les clés pour la valorisation d'une recherche scientifique à partir de l'usage du plurilinguisme comme une stratégie discursive. Nous réfléchissons aussi à la manière dont cette stratégie peut contribuer à élargir les façons de transmettre des savoirs scientifiques dans le but d'atteindre la société de manière plus efficace.

\footnotetext{
${ }^{1}$ Nous remercions Thomas de Fornel de l'Université de Bordeaux (France) pour sa contribution à la révision théorique et linguistique de ce travail.

${ }^{2}$ Le Programa de Pós-Graduação em Letras (PPGL) offre des formations de master et de doctorat dans les domaines de la linguistique et de la littérature.

3 Traduction: Ma recherche en 5 minutes.
} 


\section{Cadre théorique}

\subsection{Apports des approches plurielles à la didactique des langues}

L'émergence de nouveaux environnements sociaux-communicatifs entraine toujours des changements dans le domaine de la didactique des langues (DL), qui s'applique constamment à répondre aux besoins concrets de la communication humaine, avec toute sa variété de formes et d'usages, en cherchant les meilleures stratégies pour enseigner et faire apprendre les langues. Sur ce point, on se rend compte qu'avec le phénomène de la mondialisation, l'accélération des mouvements humains et des échanges de biens, de services, de technologies, de pratiques culturelles, etc. - a entrainé des changements dans les façons de communiquer. En ce sens, on voit dans les espaces sociaux la coexistence spontanée d'une diversité de langues et de cultures qui ont tendance à s'articuler les unes par rapport aux autres, à franchir les frontières des pays avec les personnes et leurs interactions.

Dans l'enseignement des langues, ces changements communicatifs ont été reconnus par un « multilingual turn » (Melo-Pfeifer, 2018), qui remet en cause les approches dites monolingues, voire l'idéologie du monolinguisme elle-même, c'est-à-dire l'idée que les langues doivent être étudiées de façon fermée et isolée les unes des autres, sans tenir compte de leurs connexions, de leurs contacts et de leurs points communs. La didactique du plurilinguisme établit un dialogue épistémologique avec ce nouveau paradigme et cherche à créer des stratégies efficaces d'enseignement et d'apprentissage ayant pour but de développer une compétence globale de communication chez l'apprenant, qui est à la fois plurilingue et pluriculturelle.

Si le plurilinguisme n'est pas un phénomène nouveau, car l'histoire des langues est aussi l'histoire du contact entre les langues qui a toujours existé dans un processus naturel d'hybridation, sa reconnaissance dans le domaine de la DL est plus récente. D’après Simonin et Wharton (2013, p. 16),

[...] les chercheurs qui prennent pour objet les phénomènes de contact de langues, reconnaissent pour la plupart que ce n'est que depuis la fin de la seconde guerre mondiale et à un rythme très lent que ce secteur s'est développé, pour s'intensifier et prendre son essor à la fin du XXe siècle.

Légitimée avant tout par des études sociolinguistiques, cette approche remet en question les fondements théoriques, les concepts et les démarches pédagogiques déjà cristallisés à partir du monolinguisme. Ne pas réduire l'enseignement des langues à l'enseignement d'une seule langue, ne pas juxtaposer une langue après l'autre comme des univers fermés et indépendants, ne pas percevoir une langue comme une homogénéité linguistique à l'intérieur d'elle-même (l'idée de parler une seule langue dans la même variété et de la même façon), c'est en cela que le plurilinguisme propose un regard positif sur l'hétérogénéité linguistique et culturelle de la communication humaine, dont la mondialisation fait actuellement preuve. 
Développée par le Cadre Européen Commun de Référence pour les Langues (CECR) ${ }^{4}$, puis par le Guide pour l'élaboration des politiques linguistiques éducatives en Europe ${ }^{5}$ du Conseil de l'Europe, la notion de compétence plurilingue et pluriculturelle est introduite au milieu des années 1990 (Coste, 2019), dans le but de promouvoir une éducation linguistique pour la construction d'une société européenne plus tolérante et respectueuse des différences linguistiques et culturelles entre les personnes - un point essentiel de divers projets politiques et éducatifs de l'Union européenne. Parmi les documents qui visent à organiser cette éducation, le Cadre de Référence pour les Approches Plurielles des Langues et des Cultures $(C A R A P)^{6}$ propose une définition de ce que l'on appelle les approches plurielles - l'approche interculturelle, l'éveil aux langues, l'intercompréhension entre les langues parentes et la didactique intégrée des langues - qui mettent en place des démarches didactiques reposant sur la notion de compétence plurilingue et pluriculturelle (Candelier, 2009).

Chaque approche plurielle développe de façon particulière un certain principe pédagogique : centrer la pratique des langues sur la relation à l'autre dans l'approche interculturelle, construire des relations linguistiques en s'appuyant sur les proximités entre les langues pour s'intercomprendre, chercher des points de relation entre l'enseignement de la langue maternelle et l'enseignement des autres langues à tous les niveaux d'apprentissage et dans toutes les disciplines dans la perspective de la didactique intégrée des langues, et promouvoir la diversité linguistique pour sensibiliser l'apprenant à cette pluralité du paysage linguistique, à l'éveil aux langues. Pour Melo-Pfeifer, les approches plurielles remettent en cause quatre conceptions du monolinguisme :

[...] pluralistic approaches challenge the myth of both the native speaker and of pre-available linguistic norms; they abandon the idea of the classroom being a monolingual locus of teaching and learning; and they accept that interaction does not need to occur in just one language. (MeloPfeifer, 2018, p. 199).

Dans cette perspective, on s'aperçoit que peu à peu les approches plurielles entrent dans les systèmes éducatifs en Europe et en Amérique latine. Si d'un côté la didactique du plurilinguisme a une face pédagogique essentielle, elle peut également avoir un impact positif sur les actions et les attitudes

\footnotetext{
${ }^{4}$ Conseil de L'Europe. (2001). Cadre européen de référence pour les langues : apprendre, enseigner, évaluer (CECR). Paris : Didier. Voir : https://www.coe.int/fr/web/language-policy/cefr

${ }^{5}$ Conseil de L'Europe. (2007). De la diversité linguistique à l'éducation plurilingue : Guide pour l'élaboration des politiques linguistiques éducatives en Europe. Strasbourg : Division des Politiques linguistiques. Voir : https://www.coe.int/fr/web/language-policy/from-linguisticdiversity-to-plurilingual-education-guide-for-the-development-of-language-education-policies-in-europe

${ }^{6}$ Le CARAP, du Conseil de l'Europe, est le document de référence pour une éducation plurilingue et interculturelle. Le site CARAP propose une série de documents et de supports pédagogiques qui constituent des ressources pour la mise en place des projets. Voir : https://carap.ecml.at/Accueil/tabid/3577/language/fr-FR/Default.aspx
} 
communicatives dans les espaces d'enseignement, car elles développent un certain nombre d'atouts qui peuvent mener à élargir les formes d'interaction - dans un mouvement contraire à celui d'une homogénéisation linguistique et culturelle. En ce sens, pour faire comprendre notre point de départ, nous avons situé le plurilinguisme en explicitant sa place dans la didactique des langues. Nous proposons, par la suite, une réflexion sur ses contributions communicatives notamment en ce qui concerne le milieu de l'enseignement supérieur.

\subsection{Le plurilinguisme dans la diffusion et la vulgarisation de la recherche scientifique}

Le monde actuel exige une compétence en lisibilité des discours. Cela est donc également vrai pour les individus qui transitent dans différents espaces communicationnels et cela est vrai aussi pour les institutions, notamment les universités. D'après Erazo Munoz et Vieira (2018, p. 143), « les universités sont [...] des lieux d'échange et de mobilité de personnes où les formes et les choix de communication et de contact sont motivés par des choix politiques, économiques et culturels ». Si les universités sont censées être un espace pluriel à plusieurs sens, l'hégémonie de la langue anglaise dans les pratiques communicatives de l'enseignement supérieur a souvent imposé un monolinguisme sur le plan institutionnel et relationnel, créant ainsi des relations asymétriques entre les personnes, les langues, les cultures et les savoirs (Gorovitz, Jesus et Erazo Munoz, 2020).

En ce sens, on attire l'attention sur le fait que le plurilinguisme, en tant que stratégie discursive, pourrait contribuer à élargir les compétences communicatives de la communauté scientifique, à mieux intégrer les apprenants étrangers et migrants, et à mettre en place des politiques linguistiques favorisant un véritable processus d'internationalisation des universités (Erazo Munoz, 2016). II ne s'agit pas de nier l'importance de la langue anglaise, mais de briser les hiérarchies dans le sens de la placer à côté des autres langues (Melo-Pfeifer, 2018), favorisant les contacts, l'intercompréhension et la visibilité des langues.

La grande question n'est pas de savoir quelle langue est la plus importante ou la plus parlée, mais de savoir quels outils et quelles stratégies nous pourrons utiliser dans un monde vraiment plurilingue, où toutes les langues et cultures travaillent ensemble pour l'inclusion dans l'espace de l'intégration et des nouvelles approches du savoir académique (Erazo Munoz et Vieira, 2018, p. 157).

Ce changement de paradigme suggère un effort à la fois collectif et collaboratif - qui n'est pas évident mais qui est tout à fait faisable. À titre d'exemple, nous citons le cas des Massive Open Online Courses $(M O O C)$, ce dispositif d'enseignement et d'apprentissage qui existe pour une variété de domaines disciplinaires, surtout universitaires, et qui présente un format original de diffusion des connaissances, dans lequel la dimension plurilingue joue un rôle important, notamment pour l'internationalisation des 
savoirs. Prenant compte du potentiel plurilingue des $M O O C$, des recherches récentes ${ }^{7}$ se sont consacrées à comprendre quelles seraient les possibilités linguistiques offertes par ces dispositifs et quelles seraient leurs fonctions pédagogiques, communicatives et intégratives pour la communauté de l'enseignement et de la recherche.

Dans cette même perspective, le plurilinguisme peut agir pour la diffusion et la vulgarisation de la recherche scientifique, c'est-à-dire transmettre en plusieurs langues les travaux développés en milieu universitaire de façon à améliorer l'interaction entre les scientifiques et la société, principalement en facilitant l'accès aux savoirs et le partage des connaissances. Si dans la diffusion scientifique on croit que la langue anglaise peut atteindre un public plus vaste, le fait de la placer à côté des autres langues peut au contraire - ouvrir des espaces pour la construction de nouvelles façons de véhiculer des informations et des connaissances, déconstruisant ainsi l'homogénéisation à la fois linguistique et du savoir (Grin, 2013). De ce fait, le choix des langues et leurs modes d'interaction dans la communication scientifique peuvent constituer un troisième espace de négociation du sens, un "third space » dans la perspective de Bhabha $(1990,1994)$.

Dans le contexte latino-américain, en sciences, les processus de compréhension du monde sont ancrés dans des références épistémologiques issues de l'eurocentrisme, ce qui entrave le développement des connaissances fondées sur la diversité de la pensée, de la vision du monde, de l'épistémologie (Gorovitz, Jesus et Erazo Munoz, 2020). À partir de ce contexte, plus spécifiquement au Brésil, on voit une hégémonie linguistique dans la production, la diffusion et la réception des savoirs scientifiques menée surtout par la langue anglaise, qui est devenue la deuxième langue de travail dans la plupart des universités brésiliennes. D'après les mêmes auteurs,

O Brasil, como todos os que buscam adentrar a cena da ciência mundial, enfrenta o dilema estrutural entre aceitar e reforçar a hegemonia do inglês ou buscar espaços de resistência, aliando-se a outras comunidades ditas periféricas, notadamente por meio da promoção da diversidade linguística e da difusão dos conhecimentos produzidos localmente em línguas vernáculas $^{8}$ (Gorovitz, Jesus et Erazo Munoz, 2020, p. 80).

\footnotetext{
7 Voir Stratton et Grace, 2016; Fonseca et Gajo, 2020.

8 Traduction : Le Brésil, comme tous les pays qui cherchent à entrer sur la scène scientifique mondiale, est confronté au dilemme structurel entre accepter et renforcer l'hégémonie de l'anglais ou rechercher des espaces de résistance, s'alliant avec d'autres communautés dites périphériques, notamment à travers la promotion de la diversité linguistique et la diffusion des connaissances produites localement dans les langues vernaculaires.
} 
Cependant, l'argument qui déconstruit ce dilemme dit que l'existence des discours scientifiques plurilingues se relie à la diversité et à la complexité du monde dans lequel nous vivons et élargit les possibilités de compréhension des phénomènes, car il n'y aurait pas de modèle unique pour la construction des connaissances. En ce sens, on est de plus en plus conscient des avantages que le plurilinguisme apporte au domaine scientifique, notamment dans le contexte de l'internationalisation des universités et de la mobilité universitaire. Cette prise de conscience est très importante, car elle peut déjà amener au développement des expériences institutionnelles et à un cadre de réflexion plus approfondi sur ce sujet, que ce soit en Amérique latine ou en Europe.

D'après l'optique du plurilinguisme, si les langues et les cultures peuvent être davantage présentes dans les actions de l'enseignement supérieur, telles que les publications écrites, les curriculums, les formations, les événements scientifiques, la communication universitaire, les accords internationaux, la diffusion et la vulgarisation de la science, on pourra ainsi construire un véritable milieu plurilingue et pluriculturel qui s'ouvrira à la construction diversifiée des savoirs et favorisera donc l'intégration, la coopération, la tolérance et le respect des individus dans la communauté scientifique. Sur le plan communicationnel, aspect qui nous intéresse plus particulièrement, cela apporte une dimension importante, car les enseignants-chercheurs et les étudiants auront la liberté de choisir en quelles langues ils veulent s'exprimer.

\section{L'événement "Minha pesquisa em 5 minutos $\|^{\circ}$ : enjeux et attentes}

En 2020, la crise sanitaire de la COVID-19 a créé des défis sans précédent. Depuis la période d'isolement social au Brésil (en mars 2020), la communauté de l'Université Fédérale du Paraná (UFPR) - recteurs, professeurs, personnel de l'administration et étudiants ont dû agir rapidement pour restructurer les formations, s'adapter aux nouveaux formats de cours ainsi qu'ajuster les recherches en master et en doctorat. Dans ce contexte, l'initiative de création de l'événement « Minha pesquisa em 5 minutos », par la représentation étudiante du Programa de Pós-Graduação em Letras (PPGL), a permis aux étudiants de partager leurs recherches, de faire face à la distanciation physique tout en préservant les liens de la communauté scientifique et de diffuser plus largement les investigations pour atteindre un public non spécialisé.

\footnotetext{
${ }^{9}$ La description de l'événement a été faite à partir d'une traduction libre du document de référence « MINHA PESQUISA EM 5 MINUTOS I DIRETRIZES GERAIS DO EVENTO », envoyé par courriel, par le PPGL-UFPR, le 23 juillet 2020.
} 
« Minha pesquisa em 5 minutos » a débuté au premier semestre de 2020. II s'agit d'une action pour la diffusion et la vulgarisation de la science et de la technologie, dans une langue et un format accessibles et non dans un format et un registre de langue universitaire. L'événement consiste à la publication en ligne de capsules vidéo d'environ cinq minutes, produites par les étudiants eux-mêmes dans un format préétabli, dans le but de présenter au public en général (non spécialisé) une synthèse des recherches de master/doctorat menées au sein du programme PPGL. Les vidéos sont publiées sur la chaîne officielle YouTube $^{10}$ et sur le compte Instagram ${ }^{11}$ du PPGL à n'importe quel moment et peuvent être diffusées sur d'autres plateformes, comme sur Facebook et par courriel.

La participation à l'événement est réservée aux étudiants du PPGL-UFPR, c'est-à-dire uniquement aux étudiants liés au programme PPGL au moment de la présentation. L'auteur de la vidéo a l'autonomie pour aborder le sujet comme il le veut : il peut dire pourquoi il s'intéresse à cette recherche, il peut présenter un résumé du projet dans son ensemble, il peut ne présenter qu'une partie de sa recherche, il peut recentrer la discussion sur la façon dont la recherche se rapporte à la vie quotidienne, etc. Ainsi, le participant détient tous les droits d'auteur sur la vidéo produite et est également le responsable de son contenu.

Ensuite, le PPGL-UFPR s'engage à utiliser les capsules vidéo exclusivement pour la publication et la diffusion des recherches développées dans le cadre du programme. Après la publication sur les réseaux sociaux du PPGL, l'étudiant peut aussi divulguer et partager la vidéo sur ses propres réseaux. La participation du public est limitée à la réalisation des commentaires sur les plateformes et la gestion des commentaires est suivie par le secrétariat du PPGL. Les vidéos qui font partie de cet événement ne sont pas forcément soumises à un examen préalable par les professeurs et/ou directeurs de recherche.

La fiche technique des vidéos (tournage, édition, titre de la recherche, niveau d'études de la recherche, etc.) est publiée dans la description de la vidéo sur la plateforme YouTube, facilitant ainsi la recherche de métadonnées. Cette description comprend également jusqu'à trois hashtags (\#) qui décrivent la recherche (comme des mots-clés dans un résumé). Par exemple, dans une vidéo sur la recherche « Analyse de telle ou telle traduction en portugais dans le contexte $\mathrm{X}$ », les hashtags pourraient être : \#littératurecomparée, \#traduction, etc. Ces hashtags permettent de rechercher les métadonnées, c'est-à-dire que lorsqu'une personne écrit ces mots dans un moteur de recherche comme Google, la vidéo a plus de chance d'être trouvée dans les résultats.

\footnotetext{
10 Page officielle du Programa de Pós-Graduação em Letras sur la chaîne YouTube : https://www.youtube.com/c/P\%C3\%B3sGradua\%C3\%A7\%C3\%A3oemLetrasUFPR/featured.

${ }^{11}$ Page officielle du Programa de Pós-Graduação em Letras sur Instagram : https://www.instagram.com/pgletrasufpr/?hl=pt-br.
} 
Les capsules vidéo doivent être conformes aux directives techniques suivantes :

- La vidéo doit avoir une durée d'environ cinq minutes;

- La vidéo peut être éditée, elle peut contenir des diaporamas, des éléments iconographiques, etc.;

- La vidéo n'est pas en direct, mais des interactions peuvent se produire à travers les commentaires sur YouTube et Instagram;

- La production et l'édition de la vidéo sont de responsabilité de l'auteur;

- L'auteur de la vidéo doit apparaître au moins dans la présentation et la clôture;

- Comme il s'agit d'un événement de diffusion et de vulgarisation de la science et de la technologie, l'auteur de la vidéo doit viser un public non spécialisé et utiliser un langage plus accessible;

- La vidéo doit contenir les informations suivantes : 1) présentation de l'auteur (nom, prénom, etc.); 2) titre (provisoire) de la recherche (de thèse ou de mémoire); et 3) nom du directeur ou de la directrice de mémoire ou de thèse;

- Une fois que la qualité du son et de l'image est garantie, les vidéos peuvent être tournées avec toutes les ressources souhaitées par l'auteur (caméra, téléphone portable, etc.), mais doivent être envoyées à la commission d'organisation aux formats MP4 ou MOV;

- Avant de soumettre la vidéo, l'auteur doit envoyer un e-mail à la commission d'organisation avec les informations suivantes : 1) le scénario écrit de la vidéo (le discours dans la présentation) et 2) l'autorisation du directeur ou de la directrice de mémoire ou de thèse pour la publication de son contenu.

Finalement, la participation des étudiants n'est pas obligatoire et il n'y a pas de date limite maximale ou minimale pour soumettre les vidéos. Cependant, ceux qui y participent reçoivent une certification de communication orale lors d'un événement en ligne. De plus, la représentation étudiante reste responsable de la coordination générale de l'événement, étant responsable de 1) répondre aux doutes des étudiants; 2) envoyer les vidéos au secrétariat du programme, qui prendra en charge la publication et l'organisation des vidéos sur les plateformes; et 3 ) préparer le matériel de diffusion de l'événement, comme un courriel d'invitation et une affiche pour les réseaux sociaux.

Il faut remarquer qu'au-delà de la crise sanitaire, la crise actuelle de l'éducation au Brésil, située dans un contexte de polarisation des partis politiques, entraine des discours de délégitimation de la science de la part du gouvernement en place. Pour cette raison, l'événement créé par le PPGL-UFPR peut attirer l'attention sur l'importance d'interagir avec les citoyens, de leur montrer que les recherches sont utiles à la société et même d'établir une relation de confiance entre la société et la communauté scientifique. 


\subsection{Méthodologie}

Cette étude exploratoire vise à comprendre comment le plurilinguisme peut valoriser la recherche dans un événement ou une communication scientifique. Nous essayons de répondre à cette question à partir de l'analyse d'une capsule vidéo élaborée pour l'événement « Minha pesquisa em 5 minutos » et publiée sur la chaîne officielle YouTube du PPGL-UFPR, le 28 février 2021. Le corpus a pris en compte le nombre total de capsules publiées dans le cadre de l'événement jusqu'au premier semestre 2021 et a été défini à partir de celles ayant une dimension plurilingue, c'est-à-dire celles qui intègrent deux ou plusieurs langues au lieu d'en privilégier seulement une. Ainsi, nous avons identifié une vidéo de cette nature sur un total de neuf, dont cinq sont du domaine de la linguistique et quatre de la littérature.

Tout d'abord, nous définissons cette production comme un énoncé multimodal. Cette perspective est très importante, car notamment dans les études linguistiques, lorsque l'on observe des phénomènes de communication, on a tendance à donner une place prépondérante au langage verbal (Merriam et Tisdell, 2016, p. 65). Alors que si, d'une part, l'expression humaine se fait principalement à travers le langage verbal, d'autre part, elle n'est pas enfermée dans cet univers. D'après Morilhat (2008), les actes d'intercompréhension et les interactions sociales trouvent également leur expression dans d'autres types de langage, tels que la musique, la danse, les arts visuels, la photographie, les gestes, les symboles. Pour cette raison, face à la survalorisation du langage verbal, il est crucial de remettre en question cet enfermement, surtout dans les recherches qui prennent en compte les espaces virtuels, comme la nôtre.

En ce sens, selon la définition de Bakhtin (1992), un énoncé constitue un acte de communication - unique et irremplaçable - et concerne la manière dont un discours se matérialise, qui à son tour véhicule une idée, une opinion ou une pensée. Selon Rojo (2013), qui suit la perspective bakhtinienne, l'énoncé ne peut utiliser qu'un seul langage - oral ou verbal - mais il peut aussi être multimodal, c'est-à-dire combiner plusieurs types de langage - verbal, oral, visuel, sonore, gestuel. Les énoncés multimodaux sont principalement présents dans les environnements virtuels, tels que les réseaux sociaux et les plateformes sur Internet. Dans le domaine de l'éducation, nous avons comme exemple le format des $M O O C$ - un autre type énoncé de nature multimodale. Dans notre étude, ce type d'énoncé est alors observé à partir de deux axes - le contenu discursif et la forme (les composantes de la production audiovisuelle).

Dans une perspective d'analyse qualitative et interprétative, nous décrivons le paysage linguistique (PL) de la capsule pour vérifier comment l'espace discursif est structuré. Le paysage linguistique concerne la présence de langues - sous forme d'éléments sémiotiques variés - dans un espace donné, notamment l'espace urbain. II s'agit d'un aspect sociolinguistique important qui indique, par exemple, la hiérarchie des groupes ethnolinguistiques dans des environnements multilingues. Le concept de PL a notamment été formulé par les travaux de Gorter (2006), Gorter et Shohamy (2009) et englobe aussi le « linguistic netscape " (Melo-Pfeifer et Lima-Hernandes, 2020), c'est-à-dire au-delà des espaces physiques, les espaces virtuels - tels que YouTube et Instagram, et concerne des événements temporaires - comme l'événement promu par le PPGL-UFPR. 
À partir de ces définitions, dans cette étude nous comprenons le PL comme un aspect communicationnel dont la dimension plurilingue est une stratégie métadiscursive importante, puisqu'elle est à la fois la forme et le contenu de cette production audiovisuelle. Sur le plan du contenu, nous décrivons la recherche présentée dans la capsule vidéo. Sur le plan de la forme, nous analysons le PL à partir du choix des langues et de leurs règles de participation dans la construction de l'énoncé multimodal global. Pour cela, nous les classons en langue principale, langues secondaires et langues périphériques. De plus, nous vérifions comment ces langues s'intègrent aux composantes de la vidéo, c'est-à-dire le scénario, le tournage, les sous-titres, le lettering ${ }^{12}$ et les éléments iconographiques.

Finalement, nous définissons la capsule vidéo comme une production multimodale plurilingue à fonction communicationnelle, ce qui, au sens large, constitue une prise de position significative dans le contexte de la communication scientifique, comme nous l'avons vu précédemment dans la discussion théorique de cette étude. Compte tenu de la place centrale qu'occupe l'anglais dans les pratiques communicatives de l'enseignement supérieur, y compris dans la diffusion et la vulgarisation des recherches et des savoirs, l'objectif est alors de montrer comment le plurilinguisme peut valoriser la recherche dans une communication, tout en étant une stratégie intégrative de la communauté scientifique et non scientifique.

\subsection{Analyse de la vidéo : le contenu}

La recherche que présente la capsule vidéo ${ }^{13}$ se situe dans le domaine de la linguistique appliquée, dans le Programa de Pós-Graduação em Letras de l'Université Fédérale du Paraná (PPGL-UFPR), au Brésil. Plus précisément, l'étude s'inscrit dans le cadre de deux projets d'accueil linguistique, destinés au public des migrants réfugiés, visant à l'enseignement et à l'apprentissage des langues nationales : le programme Reingresso de l'UFPR, au Brésil, et le programme de Co-Formation Étudiants-Réfugiés (Co-FormER) de I'Université Grenoble Alpes (UGA), en France. Si la maîtrise des langues nationales (le portugais brésilien et le français) est l'une des clés pour le recommencement d'une vie dans le pays d'accueil, ces projets sont extrêmement importants, car ils aident les communautés réfugiées à surmonter les barrières de communication imposées par le contexte migratoire.

Le programme Reingresso est une initiative du programme Política Migratória e Universidade Brasileira (PMUB) de l'UFPR. II a été créé en 2015 dans le but de faciliter les moyens d'accès aux formations en licence de I'UFPR aux migrants et aux réfugiés régulièrement résidents au Brésil et titulaires d'un visa humanitaire qui avaient commencé leurs études supérieures dans des établissements d'enseignement du

\footnotetext{
${ }^{12}$ Le lettering est compris comme un mélange de calligraphie et d'art graphique. II sublime des mots ou des textes courts en attirant l'attention sur eux.

${ }^{13}$ Accès à la présentation : https://www.youtube.com/watch?v=rzyT5KX3C0E.
} 
pays d'origine. L'objectif du projet est de mettre en œuvre des politiques inclusives (y compris des politiques linguistiques) qui permettraient aux étudiants non seulement de reprendre les études, mais de conclure la trajectoire universitaire.

Le Co-FormER, à son tour, s'est inspiré de l'approche multidisciplinaire développée depuis 2015 dans le PMUB-UFPR, au sein duquel existe le programme Reingresso, ainsi que d'autres projets des domaines suivants : lettres, droit, psychologie, pédagogie, histoire, sociologie, informatique et médecine. Ainsi, depuis 2019, une structure d'intégration similaire a été mise en place à l'UGA (via Co-FormER), également dans le but d'offrir un meilleur soutien à la communauté de migrants réfugiés pour leur intégration dans l'enseignement supérieur et dans la société d'accueil.

À ce jour, aucune étude publiée ne s'est consacrée à l'analyse de l'enseignement linguistique des deux projets et à la comparaison de leurs structures. II convient également de mentionner que cette recherche présentée par la capsule vidéo est en cotutelle de thèse avec l'UGA, à partir de l'accord bilatéral préexistant entre les deux universités, ce qui permet la réalisation de cette étude comparative située, d'une part, au PPGL à I'UFPR et, d'autre part, au Laboratoire de Linguistique et Didactique des Langues Étrangères et Maternelles (LIDILEM) à I'UGA.

Ainsi, dans cette étude, on part du principe que la didactique de l'intercompréhension (IC), en tant qu'approche du plurilinguisme (Candelier, 2009), peut être assimilée aux pratiques pédagogiques des projets mentionnés pour favoriser les processus d'enseignement et d'apprentissage des langues nationales. En d'autres termes, cela signifie opter pour une approche méthodologique qui valorise le mode réel d'interaction sociodiscursive des migrants dans les différentes situations de communication, que ce soit en présentiel ou en virtuel. En ce qui concerne le domaine des études, il est à noter que l'IC trouve déjà sa place dans les espaces de l'UFPR et de l'UGA, et se développe grâce aux apports de l'accord bilatéral existant entre les deux universités.

Au-delà d'une meilleure compréhension théorique et méthodologique des apports de l'intercompréhension à l'enseignement et à l'apprentissage des langues nationales, la recherche prétend aussi collaborer avec des actions pédagogiques d'accueil linguistique dans le cadre de la planification des cours, de la formation des enseignants, de l'élaboration du matériel didactique, du processus d'évaluation de l'apprentissage, etc. En outre, il y a l'intention de collaborer avec les pratiques d'internationalisation de l'enseignement supérieur (ES), qui comprennent des actions telles que : la reprise d'études par les populations migrantes, la revalidation des diplômes, la formation de la communauté universitaire migrante, la documentation en différentes langues, etc.

Devant l'usage privilégié des langues nationales dans cet espace, c'est-à-dire une tendance au monolinguisme, il existe un risque de perte d'autonomie discursive de la part des migrants qui y entrent. Pour cette raison, I'IC intégrée dans les politiques linguistiques du milieu universitaire et dans les actions d'accueil peut favoriser une participation plus démocratique des étudiants migrants. Finalement, il sera possible d'apporter une réponse au problème de l'intégration de la population migrante et réfugiée dans l'ES franco-brésilien, en couvrant plus largement les contextes européen et latino-américain. En ce sens, la recherche s'inscrit dans une perspective durable, car d'autres universités d'Europe et d'Amérique latine pourront, par la suite, profiter des résultats pour développer leurs propres projets d'accueil linguistique. 


\subsection{Analyse de la capsule vidéo : le paysage linguistique}

Tout d'abord, nous vérifions comment l'espace discursif est structuré dans la capsule vidéo. Pour cela, nous considérons la synergie existante entre le langage oral, écrit et iconographique, articulés les uns aux autres dans une configuration qui échappe à toute catégorisation fermée et qui agit vers la construction d'un seul énoncé multimodal pour véhiculer le message global. Pour cette raison, il n'y a aucun moyen de les interpréter isolément, comme s'il s'agissait d'une simple association de langages. À partir de ce constat, nous observons la dimension plurilingue de cette production, c'est-à-dire le choix des langues dans la composition de l'énoncé global, et nous concluons qu'elle intègre toutes les composantes de la production audiovisuelle - scénario, tournage, sous-titrage et lettering - à part les éléments iconographiques (logos des universités, drapeaux des pays et cartes des pays).

La capsule vidéo, de 7 minutes et 6 secondes, a été tournée à l'intérieur d'un studio, ce qui s'est avéré fondamental pour garantir la qualité esthétique de cette production. Le scénario est nécessairement l'élément le plus important à son élaboration, car il est le point de départ de toutes les autres composantes : tournage, sous-titres et lettering. En ce sens, il faut aussi remarquer que, lors du tournage, un téléprompteur a été utilisé pour faciliter la mise en scène du scénario et éviter le travail de mémorisation du texte préalablement écrit. Concernant le choix des langues - portugais brésilien, français, espagnol et anglais, il s'est fait principalement en fonction du répertoire des langues parlées par la communicatrice, c'est-à-dire son répertoire plurilingue.

Ensuite, pour la conception du sous-titrage, le texte du scénario a été traduit prioritairement en langue française. L'objectif était de garantir la diffusion de cette production auprès d'un public francophone, ce qui pourrait favoriser la perspective d'internationalisation de la recherche développée en partenariat entre la France et le Brésil. En ce sens, il convient de noter que la capsule vidéo a également été publiée sur le réseau MIRIADI ${ }^{14}$ (Mutualisation et Innovation pour un Réseau de l'Intercompréhension à Distance), avec l'objectif de diffuser la recherche à d'autres enseignants-chercheurs d'Amérique latine et d'Europe qui travaillent dans le domaine du plurilinguisme et de l'intercompréhension.

À l'observation du scénario, la construction du discours échappe au schéma classique de la communication scientifique, qui se diviserait en introduction, cadre théorique, problématique,

\footnotetext{
${ }^{14}$ Voir https://www.miriadi.net/.
} 
méthodologie, résultats attendus et conclusion. Ainsi, au lieu d'une présentation traditionnelle dans le but d'alléger le contenu théorique et de motiver l'intérêt du public récepteur non spécialisé, la progression du discours se fait par des questions/réponses, comme si on établissait une conversation informelle courante avec l'interlocuteur. De plus, les questions posées, simplifiées et en plusieurs langues, apparaissent occasionnellement sur le lettering. Voici quelques exemples :

Savez-vous ce qu'est un projet d'accueil linguistique? (français)

¿Y qué tienen que ver estos proyectos con el plurilingüismo? (espagnol)

E qual a contribuição de tudo isso para a sociedade? (portugais)

Le lettering a aussi la fonction de mettre en évidence les informations les plus importantes - telles que les noms des concepts théoriques, des universités, des projets qui font partie de l'étude et toute information clé qui doit d'être retenue par le public. Les éléments iconographiques participent à cette dynamique et ont la finalité d'illustrer le discours verbal ou d'aider à sa compréhension. Cependant, dans quelle mesure ils fonctionnent comme facilitateurs de compréhension, cela reste l'objet d'une analyse sémiotique qui échappe au sujet de notre étude.

En somme, d'après un classement des langues (tableau 1), nous pourrions dire que le portugais brésilien occupe une place centrale, suivi du français - principalement présent dans les sous-titres. La langue espagnole et la langue anglaise occupent, à leur tour, une place plus périphérique.

\section{Tableau 1}

Aperçu du paysage linguistique de la vidéo

\begin{tabular}{|c|c|c|c|}
\hline Capsule vidéo & Langue principale & Langue secondaire & $\begin{array}{c}\text { Langues } \\
\text { périphériques }\end{array}$ \\
\hline $\begin{array}{c}\text { Minha pesquisa em 5 } \\
\text { minutos }\end{array}$ & Portugais (brésilienne) & Français & $\begin{array}{c}\text { Espagnol } \\
\text { Anglais }\end{array}$ \\
\hline
\end{tabular}

La figure 1 représente la participation des langues dans l'espace discursif de la capsule vidéo. La figure est composée de quatre cercles qui, ensemble, forment le paysage linguistique de l'énoncé global. 


\section{Figure 1}

La participation des langues dans la construction de l'énoncé multimodal global.

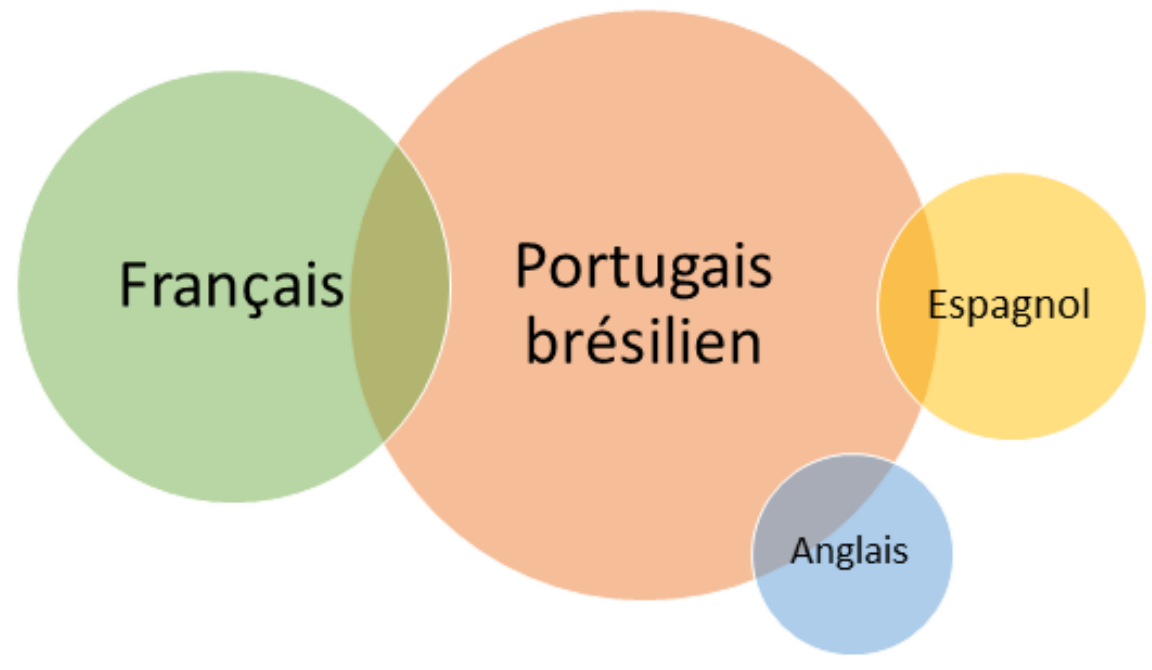

Nous avons donc le portugais brésilien dans le cercle central, qui symbolise la langue principale, le français dans le deuxième cercle à gauche pour la langue secondaire, suivi de l'espagnol dans le troisième cercle à droite et de l'anglais dans le quatrième également à droite pour les langues périphériques. L'intersection des cercles dans le cercle principal montre que le portugais brésilien est l'élément permanent tout au long de la vidéo, tandis que les autres langues apparaissent en alternance, que ce soit dans le discours oral, les sous-titres ou le lettering. Face à l'impossibilité de travailler en pourcentages pour mesurer la participation de chaque langue, la taille des cercles symbolise les degrés de participation de chaque langue dans l'espace discursif. Ainsi, nous constatons une plus grande participation du portugais brésilien, suivi du français, de l'espagnol et, enfin, de l'anglais.

Si la langue anglaise occupe souvent une place prépondérante dans la communication scientifique, il est intéressant de noter que dans cette production, elle n'a pas été mise de côté, mais a été placée dans une position hiérarchique plus horizontale avec les autres langues qui composent l'énoncé. Dans une perspective politique ancrée dans le paradigme plurilingue, cela peut montrer que l'anglais ne doit pas nécessairement être une barrière linguistique pour l'intégration de la communauté scientifique romanophone, mais peut intégrer la diversité linguistique des communications et des interactions. Par ailleurs, le choix des langues et leurs degrés de participation supposent avant tout de cibler les publics lusophones et francophones, ce qui renforce le sens dialogique de la recherche en cotutelle entre la France et le Brésil. 
Les interactions entre les langues renvoient également à la dynamique de l'intercompréhension, ce qui constitue la dimension pédagogique de cette vidéo, puisqu'elle présente une recherche sur l'IC tout en faisant appel à des stratégies intercompréhensives, c'est-à-dire des exemples pour la voir en pratique. II s'agit de montrer comment on peut tirer parti du bagage linguistique que chacun de nous possède, en le valorisant naturellement, en créant des ponts entre les langues pour faciliter l'apprentissage d'une « nouvelle » langue. Voici l'extrait du scénario et des sous-titres qui montre cet aspect :

Plus précisément, la didactique de l'intercompréhension, en tant qu'approche du plurilinguisme, peut être utilisée comme un outil pédagogique qui valorise le profil plurilingue des apprenants, leur faisant prendre conscience qu'il est possible de créer des relations entre les langues, en termes de vocabulaire, de sons, de formes, de structures et ainsi de suite.

Ces relations peuvent faciliter l'apprentissage. Ainsi, les langues connues, apprises et parlées sont utilisées par les apprenants pour améliorer leur capacité à apprendre la « nouvelle » langue.

Voulez-vous voir comment cela se passe dans la pratique?

Imaginons que je suis une migrante, récemment arrivée au Brésil et que j'ai besoin d'apprendre à me présenter en portugais. Dans mon répertoire plurilingue, il y a déjà l'espagnol, le français et l'anglais. Donc, pour dire mon nom, je connais déjà les structures :

Mi nombre es Teurra

Mon nom c'est Teurra

My name is Teurra

Je peux voir les similitudes entre les mots et les structures de ces phrases, et me rendre compte qu'en portugais c'est exactement la même chose :

Meu nome é Teurra.

Cette dimension métadiscursive du plurilinguisme - à travers l'approche intercompréhensive - constitue une action militante en sa faveur, notamment pour sa promotion comme stratégie à la fois pédagogique et communicationnelle. Sur le plan pédagogique, cela permet de démontrer ce qu'est l'intercompréhension entre langues voisines dans un processus d'intégration linguistique d'un public cible. Sur le plan communicationnel, la dimension plurilingue de cette capsule vidéo constitue une stratégie de diffusion et de vulgarisation de la recherche scientifique. Le fait d'intégrer plusieurs langues dans la communication présuppose que la réception des contenus pourra atteindre un plus grand nombre de spectateurs et ne nie pas la bonne réception du contenu chez les locuteurs de la langue principale utilisée. En ce sens, même si cela ne fait pas partie de l'objet-sujet de notre analyse, il serait intéressant de vérifier la réception de cette production à travers les données de diffusion (quantité de vues, de likes, de partages, etc.) sur la chaîne officielle YouTube et sur le compte Instagram du PPGL-UFPR, ainsi que d'observer les langues de participation dans les interactions issues des commentaires de la publication. 


\section{Le plurilinguisme comme stratégie pour valoriser la recherche scientifique : quelles clés?}

Dans cette étude exploratoire, à partir d'une démarche d'analyse qualitative et interprétative, nous avons pu observer notre production audiovisuelle, qui représente une action inédite dans le cadre de l'événement " Minha pesquisa em 5 minutos » du PPGL de l'UFPR. Bien que la dimension plurilingue n'apparaisse pas comme une exigence dans le document de référence de l'événement «MINHA PESQUISA EM 5 MINUTOS / DIRETRIZES GERAIS DO EVENTO », l'analyse réalisée permet d'avoir quelques perceptions qui montrent comment le plurilinguisme peut être utilisé comme stratégie pour valoriser la recherche dans un événement universitaire. Ainsi, à partir de notre analyse, nous mettons en évidence les facteurs qui le considèrent comme un atout potentiel dans l'élaboration d'une communication scientifique :

(1) Tenir compte du profil linguistique du public récepteur concerné par la thématique de la recherche présentée. Est-ce un public majoritairement anglophone? Germanophone? Romanophone? Effectuer le choix des langues tout en faisant attention à cet aspect.

(2) Tirer profit du répertoire linguistique du communicateur, en particulier pour la communication orale, car un travail supplémentaire de traduction peut être fait pour le sous-titrage - même dans une langue qui n'appartient pas à son répertoire individuel.

(3) Considérer que les langues peuvent intégrer toutes les composantes de la production audiovisuelle - scénario/tournage, sous-titrage et lettering, constituant ainsi l'énoncé multimodal global de la communication scientifique.

(4) Observer que le sous-titrage et le lettering plurilingue doivent cibler prioritairement les langues des espaces scientifiques où la recherche est encadrée, par exemple dans le cas d'une cotutelle de thèse, d'un accord bilatéral, d'un partenariat entre laboratoires, etc.

En somme, nous constatons que si la diversification des langues est le facteur à travers lequel la dimension plurilingue s'impose dans une communication, la gestion de l'alternance des langages - quelles langues à l'oral, quelles langues à l'écrit - est aussi un facteur important à considérer. Par ailleurs, l'importance politique du plurilinguisme est à souligner, c'est-à-dire que le fait de transmettre des connaissances en plusieurs langues favorise la création de nouveaux modes de communication scientifique et représente une action pour l'internationalisation des savoirs scientifiques.

\section{Conclusion}

Le plurilinguisme comme stratégie discursive pour la diffusion et la vulgarisation de la science et de la technologie constitue une thématique de recherche intéressante et met en lumière un type de 
communication à valoriser, notamment dans le contexte de l'internationalisation de l'enseignement supérieur et de la mondialisation. En ce sens, nous soulignons que ce travail constitue une analyse essentiellement descriptive issue d'une expérience ponctuelle de réalisation d'une capsule vidéo lors d'un contexte bien précis. Pour cette raison, cette analyse pourra être comparée ultérieurement à d'autres expériences similaires afin de constituer un cadre de réflexion plus approfondi. Cependant, nous espérons que l'expérience partagée pourra sensibiliser à l'importance des actions plurilingues vers la construction de nouveaux savoirs scientifiques dans l'enseignement supérieur et nous espérons également avoir pu contribuer à l'enrichissement des discussions de notre domaine d'études en linguistique appliquée.

\section{Liste de références}

Bakhtin, M. M. (1992). Os gêneros do discurso. Dans M. M. Bakhtin, Estética da criação verbal (G. E. M. Pereira, trad.). São Paulo: Martins Fontes.

Bhabha, H. K. (1990). The third space. Dans J. Rutherford (dir.). Identity: community, culture, difference. London: Lawrence \& Wishart.

Bhabha, H. K. (1994). The Location of Culture. London: Routledge.

Candelier, M. (2009). CARAP : Cadre de Référence pour les Approches Plurielles des Langues et des Cultures. Strasbourg, France : Conseil de l'Europe, Centre Européen pour les Langues Vivantes. https://carap.ecml.at/Portals/11/documents/CARAP Version3 F 20091019.pdf

Conseil de L'Europe. (2001). Cadre européen de référence pour les langues : Apprendre, enseigner, évaluer. Paris : Didier.

Conseil de L'Europe. (2007). De la diversité linguistique à l'éducation plurilingue : Guide pour l'élaboration des politiques linguistiques éducatives en Europe. Strasbourg : Division des Politiques linguistiques.

Coste, D. (2019). Le plurilinguisme entre variation et évaluation. Dans L. Gajo, J. Luscher, I. Racine et F. Zay (dir.). Variation, plurilinguisme et évaluation en français langue étrangère. Bern, Suisse : Peter Lang D. https://www.peterlang.com/view/title/64322

Erazo Munoz, A. (2016). Mutual intelligibility in the plurilingual context of the University of Latin-American integration: experiences, contact and plurilingual interaction. Linguistics. [thèse de doctorat, Université Grenoble Alpes]. https://tel.archives-ouvertes.fr/tel-01370807

Erazo Munoz, A., Vieira, C. (2018). Le plurilinguisme dans le cadre académique et de mobilité MERCOSUR : le cas de I'Université Fédérale d'Intégration Latino-Américaine. HETEROGLOSSIA. Quaderni di Linguaggi e Interdisciplinarità, 16, 143-162. https://riviste.unimc.it/index.php/heteroglossia/article/view/1941

Fonseca, M. et Gajo, L. (2020). Le plurilinguisme dans les MOOC : Profils d'usagers et fonctions du sous-titrage. Alsic. Apprentissage des Langues et Systèmes d'Information et de Communication, 23(2). https://doi.org/10.4000/alsic.4816

Gorovitz, S., Jesus, P. C. S. G., Erazo Munoz, A. (2020). A diversidade linguística como condição da diversidade do conhecimento científico. Revista de estudos de gestão, informação e tecnologia, 14, 74-88. http://www.revista.fatecitaqua.edu.br/index.php/regit/article/view/REGIT14-D5

Gorter, D. (dir.). 2006). Linguistic landscape: a new approach to multilingualism. Clevedon: Multilingual Matters. https://doi.org/10.21832/9781853599170

Gorter, D., Shohamy, E. (2009). Linguistic landscape: expanding the scenery. London: Routledge. https://doi.org/10.4324/9780203930960 
Grin, F. (2013). L'anglais dans l'enseignement académique : Le débat s'égare dans les clichés. Le Temps https://archive-ouverte.unige.ch/unige:38201

Melo-Pfeifer, S. (2018). The multilingual turn in foreign language education: facts and fallacies. Dans A. Bonnet, P. Siemund. Foreign language education in multilingual classrooms. John Benjamins Publishing Company.

Melo-Pfeifer, S., Lima-Hernandes, M. C. (2020). Paisagens Linguísticas: ideologias, discursos e práticas multilíngues nos espaços sociais. Domínios de Lingu@gem, 14, 1025-1059. https://doi.org/10.14393/DL44-v14n4a2020-1

Merriam, S, Tisdell, E. (2016). Qualitative research. San Francisco: Jossey-Bass.

Morilhat, C. (2008). Empire du langage ou impérialisme langagier? Lausanne : Editions Page deux.

Rojo, R. (2013). A teoria dos gêneros discursivos do Círculo de Bakhtin e os multiletramentos. Dans R. Rojo (dir.) Escol@ conectada: os multiletramentos e as TICs. São Paulo: Parábola Editorial.

Simonin, J. et Wharton, S. (dir.). (2013). Sociolinguistique du contact : Dictionnaire des termes et concepts. Lyon : ENS Éditions. https://doi.org/10.4000/books.enseditions.12366

Stratton, C. et Grace, R. (2016). Exploring Linguistic Diversity of MOOCs: Implications for International Development. Proceedings of the Association for Information Science and Technologie, 53(1). https://asistdl.onlinelibrary.wiley.com/toc/23739231/2016/53/1 